\title{
Effects of the 2014/2015 Ebola Outbreak on Malaria Management in Pregnant Women: A Comparative Study of an Ebola Affected and Unaffected Rural District of Guinea
}

\author{
Delphin Kolie $^{1, ~ *}$, Bienvenu Salim Camara ${ }^{1}$, Karifa Kourouma ${ }^{1}$, Fassou Mathias Grovogui ${ }^{1}$, \\ Jeffrey Karl Edwards ${ }^{4,7}$, Johan Van Griensven ${ }^{8}$, Rony Zachariah ${ }^{4}$ \\ ${ }^{1}$ Research Unit, National Training and Research Centre in Rural Health of Maferinyah, Forécariah, Guinea \\ ${ }^{2}$ Department of Public Health, Gamal University of Conakry, Conakry, Guinea \\ ${ }^{3}$ Woman and Child Health Research Centre, Institute of Tropical Medicine, Antwerp, Belgium \\ ${ }^{4}$ Medical Department, Doctors Without Borders Brusels, Luxembourg, Luxembourg \\ ${ }^{5}$ Department of Infection and Immunity, Luxembourg Institute of Health, Esch-sur-Alzette, Luxembourg \\ ${ }^{6}$ National Laboratory of Health, Dudelange, Luxembourg \\ ${ }^{7}$ Department of Global Health, University of Washington, Washington, United States of America \\ ${ }^{8}$ Clinical Sciences Department, Institute of Tropical Medicine, Antwerp, Belgium
} Alexandre Delamou ${ }^{1,2,3}$, Abdoul Habib Beavogui ${ }^{1,2}$, Veerle Hermans ${ }^{4}$, Claude Pierre Muller ${ }^{5,6}$,

Email address:

drdelphinkolie88@gmail.com (D. Kolie),dkolie@maferinyah.org (D. Kolie)

${ }^{*}$ Corresponding author

\section{To cite this article:}

Delphin Kolie, Bienvenu Salim Camara, Karifa Kourouma, Fassou Mathias Grovogui, Alexandre Delamou, Abdoul Habib Beavogui, Veerle Hermans, Claude Pierre Muller, Jeffrey Karl Edwards, Johan Van Griensven, Rony Zachariah. Effects of the 2014/2015 Ebola Outbreak on Malaria Management in Pregnant Women: A Comparative Study of an Ebola Affected and Unaffected Rural District of Guinea. Central African Journal of Public Health. Vol. 5, No. 4, 2019, pp. 149-155. doi: 10.11648/j.cajph.20190504.13

Received: May 15, 2019; Accepted: June 14, 2019; Published: June 29, 2019

\begin{abstract}
This study aimed to measure the effects of Ebola outbreak on antenatal care attendance and malaria management in pregnant women in Guinea. A retrospective cross-sectional study was conducted in two rural malaria-endemic health districts: on the epicentre of the West African Ebola outbreak (Guéckédou) and the other spared by Ebola (Koubia). Data were compared over similar periods of high malaria transmission before, during and after the Ebola outbreak. There were substantial declines in antenatal care visits in Guéckédou, from a monthly average number of 7,208 before the outbreak to 3,151 (57\% decrease) during and 2,843 visits $(61 \%$ decrease) after it while this indicator increased across the same periods in Koubia. In Guéckédou, the number of SP first doses provided dropped from 2,566 before the outbreak to 1,263 (51\% decrease) during and 1,010 (61\% decrease) after the Ebola outbreak $(\mathrm{P}<0.001)$ while in Koubia, the decline was by $13 \%$ and $24 \%$ during and after the outbreak from an average number of 499 doses before it $(\mathrm{P}<0.001)$. Fever cases increased by $43 \%$ and $38 \%$ during and after the outbreak respectively in Guéckédou and by $28 \%$ during and $11 \%$ after the outbreak in Koubia. Untreated malaria cases represented $11 \%$ and $12 \%$ of confirmed malaria cases during and after the outbreak respectively compared to $3 \%$ before it in Guéckédou, while in Koubia no untreated case was seen across these periods. There is need to uphold malaria services during future outbreaks.
\end{abstract}

Keywords: Malaria, Ebola, Intermittent Preventive Treatment, Guinea, Operational Research

\section{Introduction}

Malaria is among the five top killers in Africa where it claimed nearly 105,574 lives in 2016 [1,2]. In particular, during pregnancy it may be life-threatening for both the mother and the child. For example, maternal anaemia during pregnancy and low birth rate are its common consequences, especially in high transmission areas $[2,3]$.

Malaria is endemic in Guinea with a prevalence of $44 \%$ [4]. 
Its management in pregnant women has been a national health priority [4]. The World Health Organization (WHO) guideline recommends, for pregnant women, the use of Sulfadoxine-pyrimethamine (SP) as an intermittent preventive treatment (IPT) provided during antenatal care (ANC) visits [5]. The first dose of SP should be given as early as during the second trimester and subsequent doses until delivery, with at least one-month interval between the two doses [5]. In case of uncomplicated malaria in the second and third trimester, Artemisinin-based combination therapy (ACT) is recommended, while quinine is given at any gestational age for severe malaria [5]. Health indicators in 2012 showed a need for further efforts in prevention and management of malaria among pregnant women in Guinea. Nearly half of them $(49 \%)$ were diagnosed with anaemia and only $18 \%$ received the recommended two doses of SP [4]. In 2013, a new national malaria control strategy recommended the administration of 3 to 4 doses of SP as IPT instead of 2 doses [6]. Access to malaria health services and long-lasting insecticidal nets (LLINs) were also improved through the training and deployment of community health workers (CHWs) in remote areas [6].

While efforts were on-going to sustain this strategy, the country experienced the longest and largest Ebola outbreak in history, which resulted in about 2,500 deaths nationwide and caused a detrimental effect on the health system [7-12]. As a result of community fear for contracting Ebola, health workers deaths or closure of health facilities, health facility attendance for ANC dropped during the outbreak, as well as for malaria since both malaria and Ebola have fever as a common symptom [13, 14]. In addition, with the 'no touch"' policy adopted during the outbreak, health staff's compliance with malaria management guidelines could have been compromised because it requires the use of needles for blood testing and parenteral treatment $[5,15]$. Assessing possible effects of the Ebola outbreak on the management of malaria among pregnant women is vital for guiding post-Ebola interventions for both malaria and maternal health. Therefore, we evaluated the management of malaria in pregnant women before, during and after the Ebola outbreak. We are unaware of any other study assessing malaria management among pregnant women in relation to the pre, intra, and post-Ebola periods in West Africa stratified by Ebola affected and unaffected districts.

The aim of this study was to measure and compare health services attendance for ANC, fever, as well as malaria and the management of malaria in pregnant women during the high transmission period of malaria before, during and after the Ebola outbreak in two districts in rural Guinea, one affected (Guéckédou) and another one, unaffected (Koubia) by the Ebola outbreak.

\section{Methods}

\subsection{Study Design}

This was a retrospective cross-sectional study using routine programme data over three-time periods (before, during and after the Ebola outbreak).

\subsection{Study Setting}

\subsubsection{General Setting}

Guinea is located in West Africa and was the epicentre of the 2014/2015 Ebola outbreak declared by the WHO in March $14^{\text {th, }} 2014$. Guinea is bordered by six countries including Liberia and Sierra Leone which were also heavily affected by the 2014/2015 Ebola outbreak. The country is one of the poorest in the world; it has a population of 11 million $(52 \%$ women) $[4,16]$. Guinea has 33 rural districts and a coastal capital city (Conakry). The health system is tiered and includes: a primary level (413 health centres and 726 health posts), a secondary level ( 7 regional and 26 district hospitals, 8 communal health centres), and a tertiary level (3 national hospitals) [4]. There are serious shortages of human resources with only 98 health workers per 100,000 inhabitants [4].

Guinea is malaria endemic with a high transmission period during July to October [4]. In 2015, the incidence of malaria among adults was 368 cases per 1,000 population [17]. Between 2009 and 2011, there were 530 deaths of pregnant women from malaria [4]. In 2015, the WHO estimated the maternal mortality ratio at 679 per 100,000 live births in Guinea [18].

\subsubsection{Specific Setting}

The districts of Guéckédou (290,000 inhabitants) and Koubia (100,000 inhabitants) are located in the south-east and the central parts of the country, respectively [4].

Guéckédou was included in this study because it was at the epicentre of the Ebola outbreak and reported the highest mortality rate nationwide (76\%) [7]. In addition, Guéckédou belongs to the region with the highest malaria burden in the country $(61 \%)$ [4]. It has a district hospital, one communal health centre, 13 health centres and 25 health posts [4].

Koubia was randomly selected from the list of the districts spared by Ebola with higher malaria incidence $(\geq 10 \%)$ countrywide $[19,20]$. It has one district hospital, six health centres and six health posts [4].

Malaria management in pregnant women

Malaria management is in line with national guidelines which emphasize two measures to fight malaria during pregnancy [6].

First, all pregnant women are offered a LLIN at their first ANC visit as part of the preventive package and receive at least three doses of SP as IPT during the ANC from the second trimester of pregnancy until delivery.

Second, all pregnant women presenting with fever at health facility are subjected to malaria diagnostic testing. All cases of uncomplicated malaria in pregnant women should be treated with oral quinine during the first trimester of pregnancy and with ACT during the second trimester until delivery. In case of complicated malaria, pregnant women should be treated with injectable quinine or artemisinin derivatives. 


\subsection{Study Population and Timing}

We included all pregnant women presenting to the 13 primary health facilities of Guéckédou and the six of Koubia for antenatal care visits or for fever. The study period included a period before (July-December 2013), during (July-December 2014 and July-December 2015) and after the Ebola outbreak (July-December 2016).

\subsection{Data Variables and Sources of Data}

Data on malaria management at primary health care level were collected and reported monthly to the district health officer. The district health officer compiles them, including the district hospital malaria activities, and submits monthly malaria reports to the Guinean National Malaria Control Program (NMCP). Data quality checks are implemented at district level. Study variables were extracted from the monthly malaria reports at district level; they included the numbers of: ANC visits, doses of IPT, fever cases, suspected malaria cases, cases tested for malaria (either by RDT or microscopy) and outcomes, malaria cases treated.

\subsection{Data Analysis and Statistics}

Data were entered into a dedicated EpiData database (version 3.1 for entry EpiData Association, Odense, Denmark). Study variables were measured as monthly counts. Only data from the highest malaria transmission period (July-December) of the year were considered for analysis. Counts were depicted in curves before, during and after the Ebola outbreak in the affected (Guéckédou) and unaffected (Koubia) districts. Differences in monthly average numbers across the periods (before, during and after the outbreak) within the districts were assessed using the Analysis of Variances (ANOVA) and Student $t$ test while yearly proportions were compared using the Pearson's Chi-square test. The level of significance was set at a two-tailed p-value $<0.05$.

\subsection{Ethical Consideration}

Permission to carry out this study was obtained from the National Ethics Committee in Heath Research of Guinea, Conakry, Guinea. The National Ethics Committee in Health Research of Guinea approved the present study prior to any data collection. Data are fully anonymised as an aggregated malaria monthly report at district level. Only these aggregated data were used in the study, hence patient confidentiality was respected at all time and patient consent was not needed for the data collection. This research fulfilled the exemption criteria set by the Médecins Sans Frontières Ethics Review Board for a posteriori analyses of routinely collected clinical data and thus did not require MSF ERB review. It was conducted with permission from the Medical Director, Operational Centre Brussels, Médecins Sans Frontières.

\section{Results}

\subsection{Antenatal Care Visits and Malaria Prophylaxis}

Figure 1 (A) depicts the number of ANC visits and doses of SP provided as IPT to pregnant women attending the health centres in Guéckédou and Koubia districts. The number of ANC visits drastically declined in Guéckédou during and after the outbreak as compared to before the Ebola outbreak while it increased in Koubia across the same periods. In Guéckédou, the monthly average number of ANC visits was 7,208 before the outbreak then declined up to 3,151 (a 57\% decline) during the outbreak (Table 1). This continued decreasing two years after the peak of the outbreak, reaching the lowest average of 2,843 (a $61 \%$ decline) in $2016(\mathrm{P}<0.001)$. On the contrary in Koubia, the monthly average number of ANC visits increased from 1,312 before the Ebola outbreak to 1,422 (8\% increase) during and 1,438 (9\% increase) after the Ebola outbreak $(\mathrm{P}<$ 0.001).

More pronounced declines were observed in the monthly average number of SP doses provided to pregnant women during and after the Ebola outbreak in Guéckédou as compared to Koubia. In Guéckédou, the number of SP first doses provided dropped from 2,566 before the outbreak to 1,263 (51\% decrease) during and 1,010 (61\% decrease) after the Ebola outbreak $(\mathrm{P}<0.001)$ while in Koubia, the decline was by $13 \%$ and $24 \%$ during and after the outbreak from an average number of 499 doses before it $(\mathrm{P}<0.001)$. The average number of pregnant women who received at least three doses of SP in Guéckédou declined from 2,300 before the outbreak to 1,264 (45\% decrease) during the outbreak and 967 (58\% decrease) after it $(\mathrm{P}<0.001)$. In Koubia, this number declined from 608 before the outbreak to $443(27 \%$ decline) during and 339 (44\% decline) after the outbreak ( $\mathrm{P}<$ 0.001).

\subsection{Monthly Trend of Fever Cases and Their Management}

Figure 1 (B) shows the monthly trend of fever cases and suspected malaria cases among pregnant women in Guéckédou and Koubia districts. The reported monthly average numbers of these cases considerably increased during and after the outbreak in Guéckédou as compared to Koubia (Table 1). In Guéckédou, the average number of fever cases rose from 831 before the outbreak to 1,459 (43\% increase) during the outbreak and $1,348(38 \%$ increase) after the outbreak $(\mathrm{P}<0.001)$. In Koubia, the increase in this number was by $28 \%$ during and $11 \%$ after the outbreak, from an average of 77 cases before the outbreak $(\mathrm{P}<0.001)$. As for suspected malaria cases, the number increased from 84 before the outbreak to 577 (85\%) during and 356 (76\% increase) after the outbreak in Guéckédou ( $\mathrm{P}<0.001)$. In Koubia, suspected malaria cases increased by $33 \%$ during and $49 \%$ after the outbreak, from a monthly average of 39 cases before the Ebola outbreak ( $\mathrm{P}<0.001)$. 
9
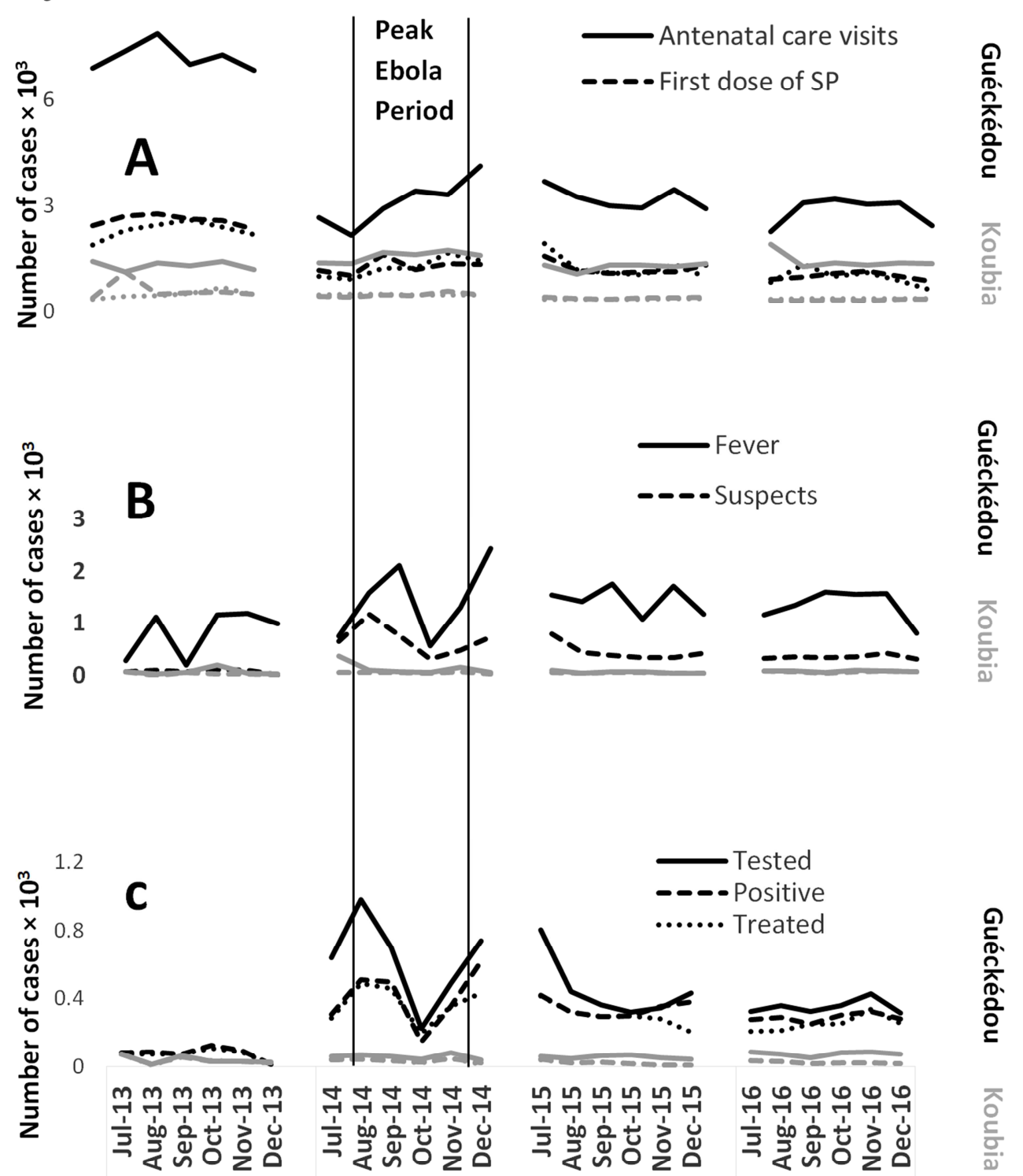

Pre-Ebola

\section{Intra-Ebola}

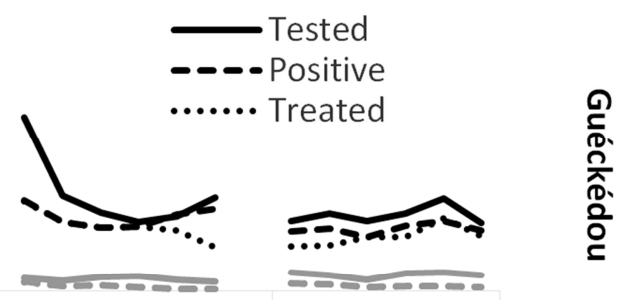

Time in months

Figure 1. Monthly trends in numbers of pregnant women utilizing the health centres of Guéckédou and Koubia health districts before (2013), during (2014-2015) and after (2016) the Ebola outbreak in Guinea.

(A) Antenatal care visits, first and at least third provision of Sulfadoxine-Pyrimethamine.

(B) All fever and suspected malaria cases.

(C) Cases tested, positive and treated for malaria.

Table 1. Monthly means ( \pm standard deviations) of antenatal care visits and malaria management indicators in Guéckédou and Koubia before (2013), during (2014-2015) and after (2016) the Ebola outbreak in Guinea.

\begin{tabular}{|c|c|c|c|c|}
\hline \multirow{2}{*}{ Variables/ Districts } & \multicolumn{3}{|c|}{ Means and Standard deviations } & \multirow{2}{*}{ P-value } \\
\hline & Before Ebola & During Ebola & After Ebola & \\
\hline \multicolumn{5}{|l|}{ Antenatal Care visits } \\
\hline Guéckédou & $7,208 \pm 391$ & $3,151 \pm 508$ & $2,843 \pm 400$ & $<0.001$ \\
\hline Koubia & $1,312 \pm 122$ & $1,422 \pm 195$ & $1,438 \pm 230$ & $<0.001$ \\
\hline \multicolumn{5}{|l|}{ First dose of SP } \\
\hline Guéckédou & $2,566 \pm 168$ & $1,263 \pm 183$ & $1,010 \pm 108$ & $<0.001$ \\
\hline Koubia & $499 \pm 111$ & $433 \pm 66$ & $379 \pm 7$ & $<0.001$ \\
\hline \multicolumn{5}{|c|}{ At least third dose of SP } \\
\hline Guéckédou & $2,300 \pm 250$ & $1,264 \pm 296$ & $967 \pm 249$ & $<0.001$ \\
\hline Koubia & $608 \pm 267$ & $443 \pm 59$ & $340 \pm 16$ & $<0.001$ \\
\hline
\end{tabular}




\begin{tabular}{|c|c|c|c|c|}
\hline \multirow{2}{*}{ Variables/ Districts } & \multicolumn{3}{|c|}{ Means and Standard deviations } & \multirow{2}{*}{ P-value } \\
\hline & Before Ebola & During Ebola & After Ebola & \\
\hline \multicolumn{5}{|l|}{ Fever cases } \\
\hline Guéckédou & $831 \pm 457$ & $1,459 \pm 534$ & $1,348 \pm 311$ & $<0.001$ \\
\hline Koubia & $77 \pm 63$ & $107 \pm 92$ & $87 \pm 16$ & $<0.001$ \\
\hline \multicolumn{5}{|l|}{ Suspected malaria } \\
\hline Guéckédou & $84 \pm 37$ & $577 \pm 261$ & $356 \pm 38$ & $<0.001$ \\
\hline Koubia & $39 \pm 24$ & $58 \pm 13$ & $77 \pm 14$ & $<0.001$ \\
\hline \multicolumn{5}{|l|}{ Cases tested for malaria } \\
\hline Guéckédou & Not available & $540 \pm 230$ & $350 \pm 42$ & $<0.001 *$ \\
\hline Koubia & $40 \pm 23$ & $57 \pm 14$ & $80 \pm 14$ & $<0.001$ \\
\hline \multicolumn{5}{|l|}{ Confirmed malaria } \\
\hline Guéckédou & $80 \pm 36$ & $376 \pm 124$ & $287 \pm 25$ & $<0.001$ \\
\hline Koubia & $37 \pm 23$ & $28 \pm 14$ & $25 \pm 7$ & $<0.001$ \\
\hline \multicolumn{5}{|c|}{ Cases treated for malaria } \\
\hline Guéckédou & $77 \pm 33$ & $336 \pm 96$ & $252 \pm 46$ & $<0.001$ \\
\hline Koubia & $37 \pm 23$ & $28 \pm 14$ & $25 \pm 7$ & $<0.001$ \\
\hline
\end{tabular}

ANOVA *Student t test.

The proportion of malaria cases among febrile pregnant women attending health centres increased from $10 \%$ before the outbreak in Guéckédou to $27 \%$ and $21 \%$ during and after it respectively $(\mathrm{P}<0.001)$ while this declined from $48 \%$ before to $27 \%$ and $28 \%$ during and after the outbreak in Koubia (Table 1).

Figure 1 (C) shows the trends of cases tested for malaria, malaria positive and cases treated with antimalarial drugs in the health centres of Guéckédou and Koubia. Of the total number of 4,507 confirmed malaria cases reported during the Ebola outbreak in Guéckédou, 4,027 were treated with antimalarial drugs; this means $480(11 \%)$ confirmed malaria cases were not treated in pregnant women attending the health centres in Guéckédou during the outbreak. The total numbers of confirmed malaria cases not treated were 210 (12\%) after and $15(3 \%)$ before the outbreak. On the opposite, in Koubia, any untreated case of confirmed malaria was reported across the same periods (table 2).

Table 2. Confirmed and untreated malaria cases among febrile pregnant women attending health centres in the health districts of Guéckédou and Koubia before (2013), during $(2014,2015)$ and after (2016) the Ebola outbreak in Guinea.

\begin{tabular}{|c|c|c|c|c|c|c|c|c|c|c|}
\hline \multirow{2}{*}{ Districts } & \multirow{2}{*}{ Variables } & \multicolumn{2}{|l|}{2013} & \multicolumn{2}{|l|}{2014} & \multicolumn{2}{|l|}{2015} & \multicolumn{2}{|l|}{2016} & \multirow{2}{*}{ P-value } \\
\hline & & count & $\%$ & count & $\%$ & count & $\%$ & count & $\%$ & \\
\hline \multirow{3}{*}{ Guéckédou } & Fever & 4,985 & & 8,795 & & 8,715 & & 8,085 & & \\
\hline & Confirmed malaria & 477 & 10 & 2,435 & 28 & 2,072 & 25 & 1,724 & 21 & $<0.001$ \\
\hline & Untreated malaria & 15 & 3 & 221 & 9 & 259 & 13 & 210 & 12 & $<0.001$ \\
\hline \multirow[b]{2}{*}{ Koubia } & Fever & 461 & & 865 & & 423 & & 524 & & \\
\hline & $\begin{array}{l}\text { Confirmed malaria } \\
\text { Untreated malaria }\end{array}$ & 222 & 48 & 210 & 24 & 122 & 29 & 147 & 28 & $\begin{array}{l}<0.001 \\
\text { N. A }\end{array}$ \\
\hline
\end{tabular}

N. A: Not applicable.

\section{Discussion}

This is the first study, to our knowledge, to compare maternal malaria services between two rural, malaria-endemic districts in Guinea during the largest known Ebola outbreak. We found significant declines in ANC visits and the use of IPT in pregnant women in the Ebola affected district (Guéckédou) compared to the unaffected one (Koubia). Additionally, increases in fever cases and suspected malaria were substantially more in Guéckédou than Koubia. Lastly, not all confirmed malaria cases in pregnant women were treated during and after the Ebola outbreak in Guéckédou.

Declines in ANC and IPT in pregnancy during the Ebola outbreak have previously been reported in Guinea and Liberia [21-23]. The decrease in health service utilization could be explained by several factors. First, the Ebola outbreak caught the Guinean community and health system unprepared. Consequently, health facilities were frequently seen as sources of Ebola transmission and communities abandoned their usage because of fear of contracting the disease [24].

It is plausible that in this context, pregnant women chose to seek antenatal care from traditional birth attendants or village midwives. The overwhelmed health system likely contributed to miscommunication of Ebola control strategies. The lack of specific treatment and high mortality rate may have had additional effects on community understanding, leading to worse misconceptions about the outbreak. Many districts in the country, including Guéckédou, experienced community resistance, sometimes violently, against the Ebola control teams in the field [25]. Such circumstances likely contributed to decrease health facility attendance as well as health services provision in affected regions.

Second, health facilities in rural areas of Guinea are underserved, with $90 \%$ of care being provided by less motivated and equipped volunteers [4]. During the Ebola outbreak, there was more need for human resources to assist in disease control and management [26]. Some healthcare 
workers might have been hired by international organizations (at higher wages) and repositioned to combat the outbreak, thus deserting their routine health service postings.

Third, the numbers of fever cases, suspected malaria and malaria tests performed were higher during and after the Ebola outbreak than before in Guéckédou. However, it remains unclear as to why these indicators increased while malaria service delivery dropped during the outbreak in Guinea [11, 13]. The reasons for the increase in malaria service delivery in our study remain speculative.

In December 2013, the NMCP and its partners scaled up combinations of malaria control strategies to improve prompt access to appropriate malaria services, particularly for populations in difficult-to-access areas [6]. For instance, in Guéckédou health district, this resulted in the recruitment and training of 622 community health workers in addition to the existing 130, to identify suspected malaria, perform rapid diagnostic tests, provide appropriate treatment and track malaria cases within the community [27]. Additionally, supplies and equipment had also been distributed to health centres to sustain efforts for this malaria control strategy.

The Ebola outbreak resulted in the decentralization of the national Ebola control team and the joint coordination of the epidemic diseases surveillance with the WHO. This likely enhanced fever notification and diagnosis particularly in Ebola affected districts.

Lastly, malaria management in pregnant women was not in line with NMCP guidelines during and after the Ebola outbreak in Guéckédou. In fact, not all pregnant women diagnosed with malaria were treated during and after the Ebola outbreak. Possibly, with the rapid spread of the outbreak, supply provisions for routine services may have shifted away, resulting in stock-outs for antimalarial drugs.

In the post-Ebola context, healthcare worker training would be vital to ensure malaria management is consistent with the NMCP guidelines, especially for vulnerable groups like pregnant women. It is also crucial to promote maternal health services for improved access to care in rural areas. Further studies are needed to understand the reasons of lower health service attendance almost one year after the end of the outbreak in Guinea.

Our study has several limitations related to data missing in the Ebola affected district of Guéckédou and the comparativeness of the health districts selected. We were unable to assess data on the number of malaria tests (by RDT or microscopy) performed in 2013. Data on RDT, ACTs, LLINs and IPTs stock-outs were not available in Guéckédou, these variables were excluded in the analysis. Data were not available in three and four health centres from August to November and December 2014 respectively, due to closure. The size of the two districts selected for this study was different and could influence variances observed. Subsequently, proportions were utilized where possible for analyses. Lastly, we could only include two districts in this study, thus the sample may not be representative of the national situation.

A strength of our study was the inclusion of all primarily health facilities of two districts with a high burden of malaria. Additionally, our data covers the highest periods of malaria transmission in Guinea and allows comparison between Ebola-affected and unaffected districts. Thus, these results might be relevant for regions with similar Ebola and malaria epidemiological characteristics within Guinea and elsewhere. Finally, the implementation and reporting of this study adhered to the internationally recognized STROBE guidelines [28].

\section{Conclusion}

The Ebola outbreak had a significant effect on the demand and offering of malaria services in pregnant women in rural Guinea. Antenatal care visits and the use of intermittent preventive treatment substantially declined in the Ebola-affected district during and after the outbreak compared to the unaffected one. More pregnant women diagnosed with malaria were untreated during and after the outbreak compared to before the outbreak in the Ebola-affected area. Improved efforts for malaria service delivery consistent with the national malaria control programme guidelines, along with community awareness-raising on the utilization of maternal health services are needed to restore malaria control in pregnant women in rural Guinea.

\section{Author Statement}

$\mathrm{DK}, \mathrm{BSC}$ and RZ were involved with conception and design of the protocol which was reviewed by, AHB, VH and AD. DK, KK and FMG were involved with acquisition of data that were analyzed by DK, BSC and KK. Data were interpreted by all authors. The first draft manuscript was written by DK, BSC, KK and FMG and reviewed by AD, AHB, VH, CPM, JKE, JVG and RZ. All authors have given approval for the final version to be published and are accountable. DK is the study guarantor of the present study.

\section{Conflict of Interest Statement}

The authors declare that they have no competing interests.

\section{Acknowledgements}

We are deeply indebted to the Médecins Sans Frontières Brussels and partners for their technical support through the Structured Operational Research and Training Initiative (SORT-IT) programme.

\section{References}

[1] WHO 2012: The leading causes of deaths in Africa. Assessed on the 12th March 2018 on: https://africacheck.org/factsheets/factsheet-the-leading-causesof-death-in-africa/.

[2] WHO 2017: World malaria report, 2017). Assessed on the 12th March 2018, on: http://www.who.int/malaria/publications/world-malaria-report $-2017 / \mathrm{en} /$ 
[3] Bardaj1 A, Sigauque B, Sanz S, et al. Impact of malaria at the end of pregnancy on infant mortality and morbidity. J Infect Dis. 2011; 203: 691-9. doi: 10.1093/infdis/jiq049.

[4] MOH. Demographic and Health Survey 2012. National Institute of statistics, Conakry, Guinee. Available: http://dhsprogram.com/publications/publication-fr280-dhs-fin al-reports.cfm (Accessed 21st February 2016).

[5] WHO. World Health Organisation. Guidelines for the treatment of Malaria. 3rd Edition ed. Geneva, Switzerland: WHO; 2015 April 2015.

[6] MOH. National malaria control programme: Malaria fighting strategic plan, 2013-2017 Conakry, Guinea.

[7] Coordination Nationale Ebola Guinee and World Health Organization, Rapport de la Situation Epidemiologique, Maladie à Virus Ebola en Guinee. Sit Rep_no 703, 2016, Coordination Nationale Ebola, Guinée: Conakry; Mars 18, 2016.

[8] Delamou A, Hammonds RM, Caluwaerts S, Utz B, Delvaux T. Ebola in Africa: beyond epidemics, reproductive health in crisis. Lancet. 2014 Dec 13; 384 (9960): 2105. doi: 10.1016/S0140-6736 (14) 62364-3.

[9] Delamou A, Ayadi AME, Sidibe S, Delvaux T et al. Effect of Ebola virus disease on maternal and child health services in Guinea: a retrospective observational cohort study, Lancet Glob Health February 22, 2017.

[10] Camara BS, Delamou A, Diro E, El Ayadi MA, Béavogui AH, Sidibé $\mathrm{S}$ et al. Influence of the 2014-2015 Ebola outbreak on the vaccination of children in a rural district of Guinea, PHA 2017; 7 (2): 161-167.

[11] Kolie D, Camara BS, Delamou A, Beavogui AH, Hermans V, Edwards KJ et al. The Ebola-effect in Guinea 2014-15: tangled trends of malaria care in children under-five, Plos One, February 2018.

[12] Kourouma K, Camara BS, Kolie D, Sidibé S, Beavogui AH, Delamou A. Analyzing the Effects of the Ebola Virus Disease Outbreak on Maternal and Child Health Services in the Health District of Beyla, Guinea Central African Journal of Public Health 2019; 5(4): 136-141.

[13] Plucinski MM, Guilavogui T, Sidikiba S, Diakite N, Diakite S, Dioubate M, et al. Effect of the Ebola-virus-disease epidemic on malaria case management in Guinea, 2014: a cross-sectional survey of health facilities. Lancet Infect Dis (2015) 15 (9): 1017-23. doi: 10.1016/S1473-3099 (15) 00061-4.

[14] Piot P, Muyembe JJ, Edmunds WJ. Ebola in West Africa: from disease outbreak to humanitarian crisis. Lancet Infect Dis. 2014.

[15] Siekmans K, Sohani S, Boima T, Koffa F, Basil L, Laaziz S. Community-based health care is an essential component of a resilient health system: evidence from Ebola outbreak in Liberia. BMC Public Health. 2017; 17 (1): 84.
[16] Recensement General de la Population Guinéenne, 31 Décembre 2014 (RGPH-3) [FRENCH].

[17] WHO. World Health Statistics 2017 : Monitoring Health for The SDGs. World Health Organization. 2017. 103 p.

[18] WHO, UNICEF, UNFPA, THE WORLD BANK, UNITED NATIONS. Trends in maternal mortality: 1990 to 2015. Estimates by WHO, UNICEF, UNFPA, The World Bank and the United Nations Population Division. Geneva; 2015.

[19] Guilavogui T, Hulle S Van, Hubbard E. Implementing Seasonal Malaria Chemoprevention in the context of Ebola in Guinea. National Malaria Control Program; CRS. Workshop; 2016.

[20] WHO. Seasonal Malaria Chemoprevention with Sulfadoxine-Pyrimethamine Plus Amodiaquine In Children. Who; f Guid. 2013; (7): 1-56.

[21] Camara BS, Delamou A, Diro E, Béavogui AH, El Ayadi AM, Sidibé S et al. Effect of the 2014/2015 Ebola outbreak on reproductive health services in a rural district of Guinea: an ecological study. Trans R Soc Trop Med Hyg. 2017 Jan 1; 111 (1): 22-29.

[22] Shannon II FQ, Horace-Kwemi E, Najjemba R, Owiti P, Edwards KJ, Shringarpure K et al. Effects of the 2014 Ebola outbreak on antenatal care and delivery outcomes in Liberia: a nationwide analysis, PHA 2017; 7 (S1): S88-S93 C 2017 The Union.

[23] Preetha 1, Kate K, Cuallah JH, Bernice D. Services for Mothers and Newborns During the Ebola Outbreak in Liberia: The Need for Improvement in Emergencies, Plos curr. 2015 April 16; 7.

[24] Ebola outbreak in Sierra Leone traced back to a single traditional healer's funeral where 14 women were infected. Available

at: http://www.dailymail.co.uk/news/article-2738904/Ebola-outbr eak-Sierra-Leone-traced-single-traditional-healer-s-funeral-14 -women-infected.html\#ixzz3FlB1t4aG. Accessed January 28, 2018.

[25] Fairhead J. Understanding social resistance to Ebola response in Guinea. Afr Stud Rev [Internet]. 2015; (April). Available from: http://www.ebola-anthropology.net/wp-content/uploads/2015/0 4/Fairhead-EbolaASRFinalSubmissionWeb.pdf

[26] WHO Emergencies preparedness, response: Key events in the WHO response to the Ebola outbreak, January 2015. Available at: http://www.who.int/csr/disease/ebola/one-year-report/who-resp onse/en/. Accessed on March 19th, 2018.

[27] Direction Préfectorale de la Santé: Rapport situationnel des agents de santé communautaire du district de Guéckédou, Mars 2017 [FRENCH].

[28] Von Elm E, Altman D G, Egger M, et al. The Strengthening the Reporting of Observational Studies in Epidemiology (STROBE) statement: guidelines for reporting observational studies. Lancet 2007; 370: 1453-1457. 